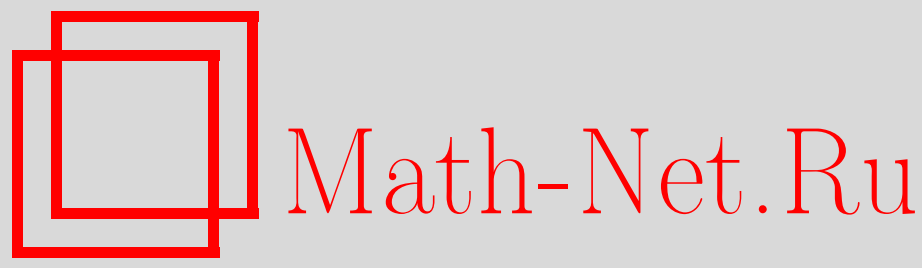

О. Н. Хакимов, $p$-Адические квазимеры Гиббса для модели Ваннименуса на дереве Кэли, ТМФ, 2014, том 179, номер 1, 13-23

DOI: https://doi.org/10.4213/tmf8536

Использование Общероссийского математического портала Math-Net.Ru подразумевает, что вы прочитали и согласны с пользовательским соглашением http://www.mathnet.ru/rus/agreement

Параметры загрузки:

IP: 3.93 .64 .190

26 апреля 2023 г., $17: 27: 58$

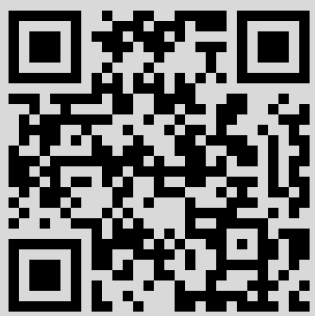




\title{
p-АДИЧЕСКИЕ КВАЗИМЕРЫ ГИББСА ДЛЯ МОДЕЛИ ВАННИМЕНУСА НА ДЕРЕВЕ КЭЛИ
}

\author{
Изучаются p-адические квазимеры Гиббса для модели Ваннименуса на де- \\ реве Кэли порядка два. Особое внимание уделено проблеме ограниченности \\ трансляционно-инвариантных $p$-адических квазимер Гиббса. Также исследова- \\ ны периодические $p$-адические квазимеры Гиббса.
}

Ключевые слова: дерево Кэли, конфигурация, квазимера Гиббса, модель Ваннименуса, трансляционно-инвариантная мера, $p$-адические числа.

DOI: $10.4213 / \operatorname{tmf} 8536$

\section{1. ВВЕДЕНИЕ}

Описание предельных мер Гиббса для данного гамильтониана является одной из основных задач в теории мер Гиббса. Полный анализ множества таких мер является весьма трудоемким. По этой причине большая часть работ по этой тематике посвящена изучению мер Гиббса на дереве Кэли [1], [2].

Известно [3]-[5], что $p$-адические модели в физике нельзя описать, используя обычную теорию вероятностей. В монографии [3] абстрактная $p$-адическая теория вероятностей была развита на основе теории неархимедовых мер. Вероятностные процессы на поле $p$-адических чисел изучались многими авторами [6]-[12]. Неархимедов аналог теоремы Колмогорова был доказан в работе [13].

В работе [8] рассматривались $p$-адические меры Гиббса для модели Изинга с четырьмя конкурирующими взаимодействиями на дереве Кэли. Доказано, что множество $p$-адических мер Гиббса состоит из единственной трансляционно-инвариантной меры Гиббса. Более того, эта мера является ограниченной. В работах [10], [11] изучались трансляционно-инвариантные $p$-адические квазимеры Гиббса для модели Поттса на дереве Кэли порядка два. Показано, что множество таких мер может состоять более чем из одного элемента. В работе [8] также рассматривались трансляционно-инвариантные $p$-адические квазимеры Гиббса для модели Ваннименуса на дереве Кэли и было доказано, что при определенных условиях существуют три трансляционно-инвариантные $p$-адические квазимеры Гиббса.

* Институт математики и информационных технологий АН РУз, Ташкент, Узбекистан. E-mail: hakimovo@mail.ru 
Настоящую работу можно считать продолжением работы [8]. Мы изучаем проблему ограниченности трансляционно-инвариантных $p$-адических квазимер Гиббса для модели Ваннименуса, а также исследуем периодические $p$-адические квазимеры Гиббса.

\section{2. ОПРЕДЕЛЕНИЯ И ФАКТЫ}

2.1. $p$-Адические числа и меры. Каждое рациональное число $x \neq 0$ может быть представлено в виде

$$
x=p^{r} \frac{n}{m}
$$

где $p$ - фиксированное простое число, $r, n \in \mathbb{Z}$, а $m$ - положительное число, взаимно простое с $n$, причем $m$ и $n$ не делятся на $p$. $p$-Адическая норма числа $x$ определяется по формуле

$$
|x|_{p}= \begin{cases}p^{-r}, & \text { если } x \neq 0 \\ 0, & \text { если } x=0 .\end{cases}
$$

Эта норма удовлетворяет сильному неравенству треугольника

$$
|x+y|_{p} \leqslant \max \left\{|x|_{p},|y|_{p}\right\}
$$

что показывает неархимедовость нормы. Из этого свойства непосредственно вытекают следующие утверждения:

1) если $|x|_{p} \neq|y|_{p}$, то $|x-y|_{p}=\max \left\{|x|_{p},|y|_{p}\right\}$;

2) если $|x|_{p}=|y|_{p}$, то $|x-y|_{p} \leqslant|x|_{p}$.

Пополнение поля рациональных чисел $\mathbb{Q}$ по $p$-адической норме приводит к полю $p$-адических чисел $\mathbb{Q}_{p}$ для каждого простого $p[14]$. Начав с поля рациональных чисел $\mathbb{Q}$, мы можем получить либо поле вещественных чисел $\mathbb{R}$, либо одно из полей $p$-адических чисел $\mathbb{Q}_{p}$ (теорема Островского).

Каждое $p$-адическое число $x \neq 0$ имеет единственное каноническое разложение

$$
x=p^{\gamma(x)}\left(x_{0}+x_{1} p+x_{2} p^{2}+\cdots\right),
$$

где $\gamma=\gamma(x) \in \mathbb{Z}$ и $x_{j}$ - целые числа такие, что $x_{0}>0$ и $0 \leqslant x_{j} \leqslant p-1$ для $j=0,1,2, \ldots[5],[14],[15]$. В этом случае $|x|_{p}=p^{-\gamma(x)}$.

Следующая теорема доказана в книге [5].

Tеорема 1. Пусть $0 \neq a=p^{\gamma(a)}\left(a_{0}+a_{1} p+\cdots\right)$, где $a_{0}>0 u 0 \leqslant a_{j} \leqslant p-1$ для $j=0,1,2, \ldots$. Уравнение $x^{2}=$ а имеет решение $x \in \mathbb{Q}_{p}$ тогда и только тогда, когда выполняются следующие условия:

1) число $\gamma($ а) четное;

2) уравнение $y^{2} \equiv a_{0}(\bmod p)$ разрешимо, если $p \neq 2, u a_{1}=a_{2}=0$, если $p=2$.

Для $a \in \mathbb{Q}_{p}$ и $r>0$ введем обозначение

$$
B(a, r)=\left\{x \in \mathbb{Q}_{p}:|x-a|_{p}<r\right\} .
$$

p-Адический логарифм определяется как ряд

$$
\log _{p}(x)=\log _{p}(1+(x-1))=\sum_{n=1}^{\infty}(-1)^{n+1} \frac{(x-1)^{n}}{n}
$$


который сходится при $x \in B(1,1) ;$-адическая экспонента определяется как

$$
\exp _{p}(x)=\sum_{n=0}^{\infty} \frac{x^{n}}{n !},
$$

этот ряд сходится при $x \in B\left(0, p^{-1 /(p-1)}\right)$.

ЛЕмма 1. Пусть $x \in B\left(0, p^{-1 /(p-1)}\right)$. Тогда

$$
\begin{gathered}
\left|\exp _{p}(x)\right|_{p}=1, \quad\left|\exp _{p}(x)-1\right|_{p}=|x|_{p}, \quad\left|\log _{p}(1+x)\right|_{p}=|x|_{p} \\
\log _{p}\left(\exp _{p}(x)\right)=x, \quad \exp _{p}\left(\log _{p}(1+x)\right)=1+x
\end{gathered}
$$

Более подробно об основах $p$-адического анализа и $p$-адической математической физики можно прочитать в монографиях [5], [14], [15].

Пусть $(X, \mathcal{B})$ - измеримое пространство, где $\mathcal{B}$ - алгебра подмножеств в $X$. Функция $\mu: \mathcal{B} \rightarrow \mathbb{Q}_{p}$ называется $p$-адической мерой, если для любого набора множеств $A_{1}, \ldots, A_{n} \in \mathcal{B}$ такого, что $A_{i} \cap A_{j}=\varnothing$ при $i \neq j$, имеет место равенство

$$
\mu\left(\bigcup_{j=1}^{n} A_{j}\right)=\sum_{j=1}^{n} \mu\left(A_{j}\right)
$$

p-Адическая мера называется вероятностной, если $\mu(X)=1$ (см. работу [13]).

2.2. Дерево Кэли. Дерево Кэли $\Gamma^{k}=(V, L)$ порядка $k \geqslant 1$ есть бесконечное дерево (граф без циклов), из каждой вершины которого выходит ровно $k+1$ ребер, $V$ - множество вершин и $L$ - множество ребер. Две вершины $x$ и $y$ называются ближайшими соседями, если существует ребро $l \in L$, соединяющее их; это записывается как как $l=\langle x, y\rangle$. Расстояние $d(x, y)$ между вершинами $x$ и $y$ равно числу ребер кратчайшего пути, соединяющего $x$ и $y$.

Пусть $x^{0}$ - фиксированная вершина. Введем обозначения

$$
W_{n}=\left\{x \in V: d\left(x, x^{0}\right)=n\right\}, \quad V_{n}=\bigcup_{m=0}^{n} W_{m}
$$

и для $x \in W_{n}$

$$
S(x)=\left\{y \in W_{n+1}: d(x, y)=1\right\} .
$$

Обычно говорят, что $S(x)$ - это множество прямых потомков элемента $x$. Две вершины $y$ и $z$ называются следующими ближайшими соседями, если существует вершина $x \in V$ такая, что $y, z \in S(x)$; это обозначается как $\rangle y, z<$.

2.3. Модель Ваннименуса. Рассмотрим р-адическую модель Ваннименуса на дереве Кэли порядка два.

Пусть $\mathbb{Q}_{p}$ - поле $p$-адических чисел и $\Phi=\{-1 ; 1\}$. Конфигурация $\sigma$ в $V$ определяется как функция $x \in V \rightarrow \sigma(x) \in \Phi$; аналогично определяются конфигурации $\sigma_{n}$ и $\sigma^{(n)}$ на $V_{n}$ и $W_{n}$ соответственно. Множество всех конфигураций на $V$ (на $V_{n}, W_{n}$ ) обозначается через $\Omega=\Phi^{V}$ (соответственно через $\Omega_{V_{n}}=\Phi^{V_{n}}, \Omega_{W_{n}}=\Phi^{W_{n}}$ ). Для конфигураций $\sigma_{n-1} \in \Omega_{V_{n}}$ и $\varphi^{(n)} \in \Omega_{W_{n}}$ положим

$$
\left(\sigma_{n-1} \vee \varphi^{(n)}\right)(x)= \begin{cases}\sigma_{n-1}(x), & \text { если } x \in V_{n-1}, \\ \varphi^{(n)}(x), & \text { если } x \in W_{n} .\end{cases}
$$

Очевидно, что $\sigma_{n-1} \vee \varphi^{(n)} \in \Omega_{V_{n}}$. 
Гамильтониан $H_{n}: \Omega_{V_{n}} \rightarrow \mathbb{Q}_{p}$ p-адической модели Ваннименуса имеет следующий вид:

$$
H_{n}(\sigma)=J_{1} \sum_{\langle x, y\rangle \in L_{n}} \sigma(x) \sigma(y)+J_{2} \sum_{\substack{>x, y<\\ x, y \in V_{n}}} \sigma(x) \sigma(y),
$$

где $J_{1}, J_{2} \in \mathbb{Q}_{p}$.

ЗАмечАниЕ 1. Модель Ваннименуса является обобщением модели Изинга, модель Изинга отвечает случаю $J_{2}=0$. Более подробно о модели Ваннименуса можно найти в книге [12].

2.4. Построение $p$-адической квазимеры Гиббса. Следуя работам [10], [11], построим $p$-адическую меру Гиббса для модели (1). Как и в классическом случае, мы рассмотрим специальный класс мер Гиббса.

Пусть $h: x \rightarrow h_{x} \in \mathbb{Q}_{p} \backslash\{0\}$ есть $p$-адическая функция на множестве $V$. Рассмотрим $p$-адическое вероятностное распределение $\mu_{h}^{(n)}$ на $\Omega_{V_{n}}$, заданное как

$$
\mu_{h}^{(n)}\left(\sigma_{n}\right)=Z_{n, h}^{-1} p^{H_{n}\left(\sigma_{n}\right)} \prod_{x \in W_{n}} h_{x}^{\sigma(x)}, \quad n=1,2, \ldots,
$$

где $Z_{n, h}$ - нормировочная константа,

$$
Z_{n, h}=\sum_{\varphi \in \Omega_{V_{n}}} p^{H_{n}(\varphi)} \prod_{x \in W_{n}} h_{x}^{\varphi(x)}
$$

Говорят, что $p$-адическое вероятностное распределение $\mu_{h}^{(n)}$ согласованно, если для всех $n \geqslant 1$ и любых $\sigma_{n-1} \in \Omega_{V_{n-1}}$

$$
\sum_{\varphi \in \Omega_{W_{n}}} \mu_{h}^{(n)}\left(\sigma_{n-1} \vee \varphi\right)=\mu_{h}^{(n-1)}\left(\sigma_{n-1}\right)
$$

В этом случае по теореме Колмогорова [13] существует единственная мера $\mu_{h}$ на $\Omega$ такая, что $\mu_{h}\left(\left\{\left.\sigma\right|_{V_{n}}=\sigma_{n}\right\}\right)=\mu_{h}^{(n)}\left(\sigma_{n}\right)$ для всех $n \in \mathbb{N}$ и любых $\sigma_{n} \in \Omega_{V_{n}}$.

ОПРЕДЕЛЕНИЕ 1. p-Адическая вероятностная мера $\mu$ называется $p$-адической квазимерой Гиббса, если существует $p$-адическая функция $h$ на $V$ такая, что

$$
\mu\left(\sigma \in \Omega:\left.\sigma\right|_{V_{n}}=\sigma_{n}\right)=\mu_{h}^{(n)}\left(\sigma_{n}\right)
$$

при всех $n \in \mathbb{N}$ и любых $\sigma_{n} \in \Omega_{V_{n}}$. Здесь $\mu_{h}^{(n)}$ определена формулами $(2),(3)$.

Заметим, что $p$-адические квазимеры Гиббса $\mu_{h}$ и $\mu_{-h}$, соответствующие функциям $h$ и $-h$, одинаковы.

Обозначим через $\mathcal{Q G}(H)$ множество всех $p$-адических квазимер Гиббса, соответствующих функциям $h=\left\{h_{x}, x \in V\right\}$. Рассмотрим гамильтониан (1) в случае $J=J_{1}=J_{2} \in \mathbb{Z}$.

Следующее утверждение доказано в работе [8]. 
УтВеРЖДЕНИЕ 1. Семейство р-адических вероятностных мер $\mu_{h}^{(n)}, n=1,2, \ldots$, удовлетворяет условию согласованности (4) тогда и только тогда, когда для любого $x \in V$ имеет место следующее соотношение:

$$
u_{x}=\frac{\theta^{2} u_{y} u_{z}+u_{y}+u_{z}+1}{u_{y} u_{z}+u_{y}+u_{z}+\theta^{2}}, \quad \theta=p^{2 J}, \quad u_{x}=h_{x}^{2}
$$

здесъ $S(x)=\{y, z\}$.

ЗАмечАниЕ 2. Вещественнозначные меры Гиббса возникают во многих проблемах теории вероятностей и статистической механики. Эти меры определяются с помощью функции экпоненты. Аналогично, p-адическая мера Гиббса определяется с помощью $p$-адической экпоненты $\exp _{p}(x)$. Но область определения и область значения функции $\exp _{p}(x)$ не очень удобна для работы. Поэтому для многих моделей, в частности для модели Изинга, существует только одна $p$-адическая мера Гиббса. Чтобы получить широкий класс $p$-адических мер Гиббса, в работе [10] было введено понятие $p$-адической квазимеры Гиббса, которая определяется с помощью функции $p^{x}$. В работах [10], [11] для модели Поттса и в работе [8] для модели Ваннименуса показано, что множество $\mathcal{Q G}(H)$ шире, чем множество всех $p$-адических мер Гиббса. Более того, $p$-адические квазимеры Гиббса могут быть неограниченными [11].

\section{3. ТРАНСЛЯЦИОННО-ИНВАРИАНТНАЯ КВАЗИМЕРА ГИББСА}

Решения уравнения (5) вида $u_{x}=u \in \mathbb{Q}_{p}$ для $x \neq x_{0}$ называются трансляционно-инвариантными. Соответствующая р-адическая квазимера Гиббса называется трансляционно-инвариантной мерой Гиббса.

Подставляя $u$ вместо $u_{x}$ для всех $x \neq x_{0}$, из уравнения (5) получим

$$
u=\frac{\theta^{2} u^{2}+2 u+1}{u^{2}+2 u+\theta^{2}} .
$$

Легко проверить, что $u_{0}=1$ является решением уравнения (6). Так как уравнение (6) является кубическим, для других решений (если они существуют) имеем формальную запись

$$
u_{1,2}=\frac{\theta^{2}-3 \pm \sqrt{\left(1-\theta^{2}\right)\left(5-\theta^{2}\right)}}{2} .
$$

Известны следующие теоремы [8].

Теорема 2. Пусть $J>0$. Тогда имеют место следующие утверждения.

1. Если $p \in\{2,3,5\}$, то существует единственная транслячионно-инвариантная р-адическая квазимера Гиббса $\mu_{h_{0}}$.

2. Пусть $p>5$ и $x_{0}$ является решением уравнения $x^{2} \equiv 5(\bmod p)$. Если уравнение $x^{2}+6 \equiv 2 x_{0}(\bmod p)$ имеет решение, то существуют три трансляционноинвариантные р-адические квазимеры Гиббса $\mu_{h_{0}}, \mu_{h_{1}}, \mu_{h_{2}}$. Здесъ $h_{0}=1, h_{1}=\sqrt{u_{1}}$, $h_{2}=\sqrt{u_{2}}$.

Теорема 3. Пусть $J<0$. Тогда существуют три транслячионно-инвариантные р-адические квазимеры Гиббса $\mu_{h_{0}}, \mu_{h_{1}}, \mu_{h_{2}}$. 


\section{1. Ограниченность трансляционно-инвариантных $p$-адических квази- мер Гиббса.}

ЛЕмма 2. Пусть $h$ является решением уравнения (5) и $\mu_{h}$ - соответствующая р-адическая квазимера Гиббса. Тогда для нормировочной константы $Z_{n, h}$ (cм. (3)) имеет место равенство $Z_{n+1, h}=A_{n, h} Z_{n, h}$, где

$$
A_{n, h}=\prod_{x \in W_{n}} a_{h}(x)
$$

ДокАЗАтЕльство. Так как $h$ является решением уравнения (5), для любой вершины $x \in V$ существует величина $a_{h}(x) \in \mathbb{Q}_{p}$ такая, что

$$
\sum_{\varphi \in \Omega_{W_{n+1}}} p^{J(\sigma(x)(\varphi(y)+\varphi(z))+\varphi(y) \varphi(z))} h_{y}^{\varphi(y)} h_{z}^{\varphi(z)}=a_{h}(x) h_{x}^{\sigma(x)}
$$

здесь $S(x)=\{y, z\}$ и $\sigma \in \Omega_{V_{n}}$. Отсюда

$$
\begin{aligned}
\prod_{x \in W_{n}} \sum_{\varphi \in \Omega_{W_{n}+1}} p^{J(\sigma(x)(\varphi(y)+\varphi(z))+\varphi(y) \varphi(z))} h_{y}^{\varphi(y)} h_{z}^{\varphi(z)}= \\
=\prod_{x \in W_{n}} a_{h}(x) h_{x}^{\sigma(x)}=A_{n, h} \prod_{x \in W_{n}} h_{x}^{\sigma(x)},
\end{aligned}
$$

где $A_{n, h}$ определены формулой (7). Из соотношений (2) и (9) получим

$$
\begin{aligned}
\sum_{\sigma \in \Omega_{V_{n}}} \sum_{\varphi \in \Omega_{W_{n}+1}} \mu_{h}^{(n+1)}(\sigma \vee \varphi) & =\sum_{\sigma \in \Omega_{V_{n}}} \sum_{\varphi \in \Omega_{W_{n+1}}} \frac{1}{Z_{n+1, h}} p^{H(\sigma \vee \varphi)} \prod_{x \in W_{n+1}} h_{x}^{\varphi(x)}= \\
& =\frac{A_{n, h}}{Z_{n+1, h}} \sum_{\sigma \in \Omega_{V_{n}}} p^{H(\sigma)} \prod_{x \in W_{n}} h_{x}^{\sigma(x)}=\frac{A_{n, h}}{Z_{n+1, h}} Z_{n, h}=1 .
\end{aligned}
$$

Лемма доказана.

Пусть $h$ является решением уравнения (5). Найдем коэффициент $a_{h}(x)$, соответствующий этому $h$. Фиксируем точку $x \in V$ и перепишем равенство (8) для случаев $\sigma(x)=1$ и $\sigma(x)=-1$. Имеем для $\sigma(x)=1$

$$
p^{3 J} h_{y} h_{z}+p^{-J} h_{y}^{-1} h_{z}+p^{-J} h_{y} h_{z}^{-1}+p^{-J} h_{y}^{-1} h_{z}^{-1}=a(x) h_{x},
$$

а для $\sigma(x)=-1$

$$
p^{-J} h_{y} h_{z}+p^{-J} h_{y}^{-1} h_{z}+p^{-J} h_{y} h_{z}^{-1}+p^{3 J} h_{y}^{-1} h_{z}^{-1}=a(x) h_{x}^{-1} .
$$

Перемножив эти равенства, получим

$$
a_{h}(x)=\frac{\sqrt{\left(p^{4 J} h_{y}^{2} h_{z}^{2}+h_{y}^{2}+h_{z}^{2}+1\right)\left(h_{y}^{2} h_{z}^{2}+h_{y}^{2}+h_{z}^{2}+p^{4 J}\right)}}{p^{J} h_{y} h_{z}} .
$$

Для трансляционно-инвариантных решений $h$ формула (10) имеет вид

$$
a_{h}=\frac{\sqrt{\left(p^{4 J} h^{4}+2 h^{2}+1\right)\left(h^{4}+2 h^{2}+p^{4 J}\right)}}{p^{J} h^{2}} .
$$




\subsection{1. Случай $J>0$.}

Лемма 3. Для любой конфигураиии $\sigma \in \Omega_{V_{n}} u n \geqslant 1$ имеет место неравенство

$$
\left|p^{H_{n}(\sigma)}\right|_{p} \leqslant p^{J\left(2^{n}-1\right)} .
$$

ДокАЗАтЕЛЬство. Легко убедиться, что $H_{n}(\sigma) \geqslant-J\left(2^{n}-1\right)$. Заметим, что гамильтониан достигает своего минимума, например конфигурация $\sigma \in \Omega_{V_{n}}$, определенная как $\sigma(y) \sigma(z)=-1$ при всех $x \in V_{n-1}$ (здесь $S(x)=\{y, z\}$ ), дает минимальное значение гамильтониана.

ЛЕмма 4. Справедливы равенства $\left|h_{0}\right|_{p}=\left|h_{1}\right|_{p}=\left|h_{2}\right|_{p}=1$.

ДоказАтельство. Очевидно, что $\left|h_{0}\right|_{p}=1$, так как $h_{0}=1$. В силу теоремы 2 решения $h_{1}, h_{2}$ могут существовать лишь только при $p>5$. Более того, в силу свойства 1 р-адической нормы (см. п. 2.1) имеем

$$
\left|h_{1}\right|_{p}=\left|\sqrt{\frac{p^{4 J}-3+\sqrt{p^{8 J}-6 p^{4 J}+5}}{2}}\right|_{p}=|\sqrt{2 \sqrt{5}-6}|_{p}=1 .
$$

Аналогично проверяется, что $\left|h_{2}\right|_{p}=1$.

Лемма 5. Для нормировочных констант $Z_{n, h_{i}}, i=0,1,2$, имеют место следующие равенства:

$$
\left|Z_{n, h_{1}}\right|_{p}=\left|Z_{n, h_{2}}\right|_{p}=p^{J\left(2^{n}-2\right)}, \quad\left|Z_{n, h_{0}}\right|_{p}= \begin{cases}p^{J\left(2^{n}-2\right)}, & \text { если } p \neq 3 \\ p^{(J-1)\left(2^{n}-2\right)}, & \text { если } p=3\end{cases}
$$

ДокАзАтЕльство. Докажем утверждение леммы для решения $h_{1}$. Из представления (11) имеем

$$
\begin{aligned}
\left|a_{h_{1}}\right|_{p} & =\left|\frac{\sqrt{\left(p^{4 J} h_{1}^{4}+2 h_{1}^{2}+1\right)\left(h_{1}^{4}+2 h_{1}^{2}+p^{4 J}\right)}}{p^{J} h_{1}^{2}}\right|_{p}= \\
& =\left|p^{-J} \sqrt{(2 \sqrt{5}-4)(\sqrt{5}+1)}\right|_{p}=\left|p^{-J} \sqrt{6-2 \sqrt{5}}\right|_{p}=p^{J} .
\end{aligned}
$$

Далее, так как $Z_{n, h}=a_{h}^{\left|V_{n-1}\right|}$ и $\left|V_{n-1}\right|=2^{n}-2$, мы получаем

$$
\left|Z_{n, h_{1}}\right|_{p}=p^{J\left(2^{n}-2\right)} \text {. }
$$

Аналогично проверяется, что $\left|Z_{n, h_{2}}\right|_{p}=p^{J\left(2^{n}-2\right)}$.

Докажем утверждение леммы для решения $h_{0}$. Так как $h_{0}=1$, из (11) получим

$$
\left|a_{h_{0}}\right|_{p}=\left|\frac{\sqrt{\left(p^{4 J}+3\right)\left(3+p^{4 J}\right)}}{p^{J}}\right|_{p}=\left|3 p^{-J}\right|_{p}= \begin{cases}p^{J}, & \text { если } p \neq 3 \\ p^{J-1}, & \text { если } p=3 .\end{cases}
$$

Отсюда

$$
\left|Z_{n, h_{0}}\right|_{p}= \begin{cases}p^{J\left(2^{n}-2\right)}, & \text { если } p \neq 3 \\ p^{(J-1)\left(2^{n}-2\right)}, & \text { если } p=3\end{cases}
$$


Теорема 4. Справедливъ следующие утверждения.

1. Если $p \neq 3$, то все транслячионно-инвариантные р-адические квазимеры Гиббса являются ограниченными.

2. Если $p=3$, то существует единственная трансляционно-инвариантная р-адическая квазимера Гиббса $\mu_{h_{0}}$, причем она является неограниченной.

ДокАЗАТЕЛЬСТво. Пусть $p \neq 3$. В этом случае в силу леммы 5 мы имеем $\left|Z_{n, h_{i}}\right|_{p}=p^{J\left(2^{n}-2\right)}$ для $i=0,1,2$. В силу лемм 3 и 4 для любой конфигурации $\sigma \in \Omega_{V_{n}}$ и всякого $n=1,2, \ldots$

$$
\left|\mu_{h_{i}}^{(n)}(\sigma)\right|_{p}=\left|\frac{p^{H_{n}(\sigma)} \prod_{x \in W_{n}} h_{i}^{\sigma(x)}}{Z_{n, h_{i}}}\right|_{p} \leqslant \frac{p^{J\left(2^{n}-2\right)}}{p^{J\left(2^{n}-2\right)}}=1, \quad i=0,1,2 .
$$

Это означает, что в случае $p \neq 3$ все трансляционно-инвариантные $p$-адические квазимеры Гиббса $\mu_{h_{i}}, i=0,1,2$, ограничены.

Пусть $p=3$. В этом случае в силу теоремы 2 существует единственная трансляционно-инвариантная $p$-адическая квазимера Гиббса $\mu_{h_{0}}$. Покажем, что она неограничена. Определим конфигурацию $\sigma$ следующим образом: $\sigma(y) \sigma(z)=-1$ при всех $x \in V_{n-1}$ (здесь $\left.S(x)=\{y, z\}\right)$. Тогда в силу лемм 4 и 5 для нормы меры $\mu_{h_{0}}$ в этой конфигурации имеем

$$
\left|\mu_{h_{0}}^{(n)}(\sigma)\right|_{p}=\left|\frac{p^{H_{n}(\sigma)} \prod_{x \in W_{n}} h_{0}}{Z_{n, h_{0}}}\right|_{p}=\frac{p^{J\left(2^{n}-2\right)}}{p^{(J-1)\left(2^{n}-2\right)}}=p^{2^{n}-2} .
$$

Отсюда $\left|\mu_{h_{0}}^{(n)}(\sigma)\right|_{p} \rightarrow \infty$ при $n \rightarrow \infty$.

3.1.2. Случай $J<0$.

ЛЕмма 6. Для любой конфигураиии $\sigma \in \Omega_{V_{n}} u n \geqslant 1$ имеет место неравенство

$$
\left|p^{H_{n}(\sigma)}\right|_{p} \leqslant p^{-J\left(3 \cdot 2^{n}-5\right)} .
$$

ДокАЗАТЕЛьство. Заметим, что гамильтониан достигает своего минимума на конфигурации $\sigma \in \Omega_{V_{n}}$, которая принимает значение единица для всех $x \in V_{n}$.

Лемма 7. Справедливы равенства $\left|h_{0}\right|_{p}=1,\left|h_{1}\right|_{p}=p^{-2 J},\left|h_{2}\right|_{p}=p^{2 J}$.

ДоказАтельство. Очевидно, что $\left|h_{0}\right|_{p}=1$. Для нормы $h_{1}$ имеем

$$
\left|h_{1}\right|_{p}=\left|p^{2 J} \sqrt{\frac{1-3 p^{-4 J}+\sqrt{1-6 p^{-4 J}+5 p^{-4 J}}}{2}}\right|_{p}=p^{-2 J} .
$$

Так как $h_{i}=\sqrt{u_{i}}, i=1,2$, и $u_{1} u_{2}=1$, получаем $\left|h_{2}\right|_{p}=p^{2 J}$.

ЛЕмма 8. Для нормировочных констант $Z_{n, h_{i}}, i=0,1,2$, имеют место следующие равенства:

$$
\left|Z_{n, h_{1}}\right|_{p}=\left|Z_{n, h_{2}}\right|_{p}=p^{-J\left(5 \cdot 2^{n}-10\right)}, \quad\left|Z_{n, h_{0}}\right|_{p}=p^{-J\left(3 \cdot 2^{n}-6\right)} .
$$


ДокАЗАТЕльство. В силу леммы 7 имеем $h_{1}=p^{2 J} \varepsilon$, где $|\varepsilon|_{p}=1$. Следовательно,

$$
\left|a_{h_{1}}\right|_{p}=\left|\frac{\sqrt{\left(p^{12 J} \varepsilon^{4}+2 p^{4 J} \varepsilon^{2}+1\right)\left(p^{8 J} \varepsilon^{4}+2 p^{4 J} \varepsilon^{2}+p^{4 J}\right)}}{p^{5 J} \varepsilon^{2}}\right|_{p}=p^{-5 J} .
$$

Отсюда

$$
\left|Z_{n, h_{1}}\right|_{p}=p^{-J\left(5 \cdot 2^{n}-10\right)}
$$

Аналогично проверяются равенства $\left|Z_{n, h_{2}}\right|_{p}=p^{-J\left(5 \cdot 2^{n}-10\right)}$ и $\left|Z_{n, h_{0}}\right|_{p}=p^{-J\left(3 \cdot 2^{n}-6\right)}$.

Теорема 5. Все трансляционно-инвариантные р-адические квазимеры Гиббса ограничены.

ДокАЗАТЕЛьСтво следует из лемм 6-8.

\section{4. ПЕРИОДИЧЕСКАЯ $p$-АДИЧЕСКАЯ КВАЗИМЕРА ГИББСА}

Рассмотрим следующее уравнение:

$$
u=f(f(u)), \quad \text { где } \quad f(u)=\frac{\theta^{2} u^{2}+2 u+1}{u^{2}+2 u+\theta^{2}} .
$$

Заметим, что множество решений этого уравнения содержит решения уравнения $u=f(u)$. Нас интересуют только периодические (не являющиеся трансляционноинвариантными) меры, поэтому рассмотрим уравнение

$$
\frac{f(f(u))-u}{f(u)-u}=0
$$

из которого получим

$$
\theta^{2} u^{2}+\left(\theta^{2}+1\right) u+\theta^{2}=0
$$

Если существует $\sqrt{1+2 \theta^{2}-3 \theta^{4}}$ в $\mathbb{Q}_{p}$, то величины

$$
u_{3,4}=\frac{-1-\theta^{2} \pm \sqrt{1+2 \theta^{2}-3 \theta^{4}}}{2 \theta^{2}}
$$

являются решениями уравнения (12). Положим $D(\theta)=1+2 \theta^{2}-3 \theta^{4}$. Сначала мы проверим, что в $\mathbb{Q}_{p}$ существует $\sqrt{D(\theta)}$, а затем покажем, что существуют числа $\sqrt{u_{3}}$ и $\sqrt{u_{4}}$. Заметим, что из существования одного из них вытекает существование второго. Имеем по теореме Виета $u_{3} u_{4}=1$. При $\sqrt{u_{3}} \in \mathbb{Q}_{p}$ отсюда получаем, что $\sqrt{u_{4}} \in \mathbb{Q}_{p}$.

ЗАмЕчАниЕ 3. Поскольку существование одного из чисел $\sqrt{u_{1}}$ или $\sqrt{u_{2}}$ влечет существование другого, мы заключаем, что либо не существует 2-периодическая p-адическая квазимера Гиббса, либо существуют две 2-периодические $p$-адические квазимеры Гиббса. 
4.1. Случай $J>0$. В этом случае в силу теоремы 1 существует $\sqrt{D(\theta)}$ для любого простого числа $p$. Теперь проверим существование $\sqrt{u_{3}}$ в $\mathbb{Q}_{p}$.

Пусть $p=2$. Тогда имеем

$$
\begin{aligned}
u_{3} & =\frac{-1-2^{4 J}+\sqrt{1+2^{4 J+1}-3 \cdot 2^{8 J}}}{2^{4 J+1}}= \\
& =\frac{-1-2^{4 J}+1+2+2^{2}+\cdots}{2^{4 J+1}}=2^{-4 J}(1+2+\cdots) .
\end{aligned}
$$

Из теоремы 1 следует, что $\sqrt{u_{3}}$ не существует в $\mathbb{Q}_{p}$.

Пусть $p \neq 2$. Тогда

$$
\begin{aligned}
u_{3} & =\frac{-1-p^{4 J}+\sqrt{1+2 p^{4 J}-3 p^{8 J}}}{2 p^{4 J}}= \\
& =\frac{-1-p^{4 J}+1+p^{2 J}+\cdots}{2 p^{4 J}}=\frac{1+a_{1} p^{J}+a_{2} p^{2 J}+\cdots}{2 p^{2 J}} .
\end{aligned}
$$

Отсюда видно, что $\sqrt{u_{3}}$ существует тогда и только тогда, когда существует $\sqrt{2}$. Таким образом, мы доказали следующую теорему.

Теорема 6. Пусть существует $\sqrt{2}$ в $\mathbb{Q}_{p}$, тогда для модели (1) существуют две 2-периодические р-адические квазимеры Гиббса $\mu_{h_{3}} u \mu_{h_{4}}$. Здесъ $h_{i}=\sqrt{u_{i}}, i=3,4$.

4.2. Случай $J<0$. В этом случае $|\theta|_{p}>1$. Положим

$$
D(\theta)=\theta^{4}\left(-3+2 \theta^{-2}+\theta^{-4}\right) .
$$

Видно, что $\sqrt{D(\theta)}$ существует тогда и только тогда, когда существует $\sqrt{-3}$. Заметим, что для простых чисел $p \leqslant 19$ значение $\sqrt{D(\theta)}$ существует только при $p=7,13$.

Теорема 7. Справедливы следующие утверждения.

1. Если $p \in\{2,3\}$, то не существует периодическая р-адическая квазимера Гиббса.

2. Пусть $p>3$. Если уравнение $x^{2}+3 \equiv 0(\bmod p)$ не имеет решения в $\mathbb{Q}_{p}$, то не существует периодическая р-адическая квазимера Гиббса.

3. Пусть $p>3$ и число $x_{0}$ является решением уравнения $x^{2}+3 \equiv 0(\bmod p)$. Тогда существуют две 2-периодические р-адические квазимеры Гиббса в том и только том случае, когда уравнение $x^{2}-2 x_{0}+2 \equiv 0(\bmod p)$ разрешимо в $\mathbb{Q}_{p}$.

ДокАзАтельство. Так как $\sqrt{D(\theta)}$ существует тогда и только тогда, когда существует $\sqrt{-3}$, мы можем ограничиться случаем, когда уравнение $x^{2}+3 \equiv 0(\bmod p)$ разрешимо в $\mathbb{Q}_{p}$. Заметим, что $\sqrt{-3} \notin \mathbb{Q}_{p}$ при $p \leqslant 3$.

Пусть $p>3$ и число $x_{0}$ является решением уравнения $x^{2}+3 \equiv 0(\bmod p)$. Тогда

$$
u_{3}=\frac{-1-p^{4 J}+\sqrt{1+2 p^{4 J}-3 p^{8 J}}}{2 p^{4 J}}=\frac{x_{0}-1+p^{-4 J} \varepsilon}{2}, \quad|\varepsilon|_{p} \leqslant 1 .
$$

Отсюда с учетом теоремы 1 следует, что $\sqrt{u_{3}}$ существует тогда и только тогда, когда уравнение $x^{2}-2 x_{0}+2 \equiv 0(\bmod p)$ разрешимо.

В силу замечания 3 существуют две 2-периодические $p$-адические квазимеры Гиббса в том и только том случае, когда уравнение $x^{2}-2 x_{0}+2 \equiv 0(\bmod p)$ разрешимо в $\mathbb{Q}_{p}$. 
Благодарности. Автор благодарен У. А. Розикову и Ф. М. Мухамедову за полезные советы и ряд замечаний.

\section{Список литературы}

[1] P. M. Bleher, J. Ruiz, V. A. Zagrebnov, J. Statist. Phys., 79:1-2 (1995), 473-482.

[2] Х.-О. Георги, Гиббсовские меры и фазовые переходь, Мир, М., 1992.

[3] A. Yu. Khrennikov, Non-Archimedean Analysis: Quantum Paradoxes, Dynamical Systems and Biological Models, Mathematics and Its Applications, 427, Kluwer, Dordrecht, 1997.

[4] E. Marinari, G. Parisi, Phys. Lett. B, 203:1-2 (1988), 52-54.

[5] В. С. Владимиров, И. В. Волович, Е. Н. Зеленов, p-Адический анализ и математическая физика, Физматлит, М., 1994.

[6] S. Albeverio, W. Karwowski, Stochastic Processes Appl., 53:1 (1994), 1-22.

[7] K. Yasuda, Osaka J. Math., 37:4 (2000), 967-985.

[8] O. N. Khakimov, p-Adic Numbers Ultrametric Anal. Appl., 5:3 (2013), 194-203.

[9] У. А. Розиков, О.Н. Хакимов, ТМФ, 175:1 (2013), 84-92.

[10] F. M. Mukhamedov, Math. Phys. Anal. Geom., 16:1 (2013), 49-87.

[11] F. M. Mukhamedov, p-Adic Numbers Ultrametric Anal. Appl., 2:3 (2010), 241-251.

[12] U. A. Rozikov, Gibbs Measures on Cayley Trees, World Sci., Singapore, 2013.

[13] Н. Н. Ганиходжаев, Ф. М. Мухаммедов, У. А. Розиков, Узб. матем. журн., 4 (1998), $23-29$.

[14] Н. Коблиц, р-Адические числа, р-адический анализ и дзета-функиии, Мир, М., 1981.

[15] W.H. Schikhof, Ultrametric Calculus, Cambridge Studies in Advanced Mathematics, 4, Cambridge Univ. Press, Cambridge, 1984.

Поступила в редакцию 18.10.2013 